\title{
Characterization of menstrual stem cells: angiogenic effect, migration and hematopoietic stem cell support in comparison with bone marrow mesenchymal stem cells
}

Francisca Alcayaga-Miranda 1,2, Jimena Cuenca 1,2, Patricia Luz-Crawford²,3, Carolina Aguila-Díaz , Ainoa Fernandez², Fernando E Figueroa ${ }^{1}$ and Maroun Khoury ${ }^{1,2^{*}}$

\begin{abstract}
Introduction: Stem cells isolated from menstrual fluid (MenSCs) exhibit mesenchymal stem cell (MSCs)-like properties including multi-lineage differentiation capacity. Besides, menstrual fluid has important advantages over other sources for the isolation of MSCs, including ease of access and repeated sampling in a noninvasive manner. Such attributes allow the rapid culture of MenSCs in numbers that are sufficient for therapeutical doses, at lower cell passages.

Methods: In this study, we advance the characterization of MenSC populations in comparison to bone marrow derived mesenchymal stem cells (BM-MSCs) with regards to proliferation, lineage differentiation, migration potential, secretion profile and angiogenic properties in vitro and in a matrigel plug assay in mice. We additionally tested their ability to support hematopoietic stem cell (HSC) expansion in vitro.

Results: The phenotypic analysis of MenSCs revealed a profile largely similar to the BM-MSCs with the exception of a higher expression of the adhesion molecule CD49a (alpha1-integrin). Furthermore, the fibroblast colony forming units (CFU-F) from MenSCs yielded a 2 to 4 fold higher frequency of progenitors and their in vitro migration capacity was superior to BM-MSCs. In addition, MenSCs evidenced a superior paracrine response to hypoxic conditions as evidenced by the secretion of vascular endothelial growth factor and basic fibroblast growth factor and also improved angiogenic effect of conditioned media on endothelial cells. Furthermore, MenSCs were able to induce angiogenesis in a matrigel plug assay in vivo. Thus, an 8-fold increase in hemoglobin content was observed in implanted plugs containing MenSCs compared to BM-MSCs. Finally, we demonstrated, for the first time, the capacity of MenSCs to support the ex-vivo expansion of HSCs, since higher expansion rates of the CD34 + CD133+ population as well as higher numbers of early progenitor (CFU-GEMM) colonies were observed in comparison to the BM source.
\end{abstract}

Conclusions: We present evidence showing superiority of MenSCs with respect to several functional aspects, in comparison with BM-MSCs. However, the impact of such properties in their use as adult-derived stem cells for regenerative3 medicine remains to be clarified.

\footnotetext{
* Correspondence: mkhoury@uandes.c

'Laboratory of Nano-Regenerative Medicine, Faculty of Medicine, Universidad de Los Andes, Santiago, Chile

${ }^{2}$ Cells for Cells, Santiago, Chile

Full list of author information is available at the end of the article
} 


\section{Introduction}

Mesenchymal stem cells (MSCs) are self-renewing progenitor cells with the capacity to differentiate into various cell types under specific conditions. Adult stem cells derived from different sources, including bone marrow, adipose tissue or post-natal tissues, such as umbilical cord and placenta, have been shown to possess regenerative, antiinflammatory or immunoregulatory potential in a variety of diseases. The limitation of their clinical use resides in the invasiveness of the extraction methods and in some cases their limited proliferative capacity. Furthermore, diverse MSCs sources are known to display distinct functional properties that might contribute to specific therapeutic effects [1].

A study published in 2007, was the first to identify and characterize a new source of stem cells within menstrual fluid. It showed that menstrual-derived stem cells (MenSCs) are rapidly expanded and differentiated under standard laboratory conditions [2]. There is growing interest in their clinical potential since they display a high proliferation rate, are multipotent and obtainable in a periodic and noninvasive manner, devoid of the biological and ethical issues concerning other stem cell types [2-5]. Recent evidence suggests that MenSCs are positive for several MSCs markers, including CD90, CD29, CD105, and CD73, and also remain negative for hematopoietic cell markers, such as CD34, CD45 and CD133. Some reports have demonstrated the expression of embryonic markers and pluripotent intracellular cell markers, such as OCT-4, c-kit and SSEA-4, not found on MSCs from other sources, although these findings have also been disputed, even in cells isolated and cultured under comparable conditions [2-7].

A detailed characterization of the MenSCs is a prerequisite for head-to-head comparisons with related cell types isolated from other sources, especially the most extensively studied bone marrow derived mesenchymal stem cells (BM-MSCs) that are already in clinical use for specific applications. Since to date there are no 'potency' tests available for MSCs, a thorough cell characterization is still a prerequisite prior to the use of a new cell type in clinical applications under safe and effective conditions.

Several studies related to the paracrine angiogenic effects of MSCs have been published since the therapeutic benefits of angiogenesis in different disease models are well-known [8-10]. Meng et al. showed that angiogenic factors (ANG-2, vascular endothelial growth factor (VEGF), HGF, and EGF) were constitutively present in the culture media of MenSCs as opposed to cord blood MSCs. The supportive effect of MSCs for hematopoiesis has been well documented in many studies including our published work [11], pointing to the possibility of expanding human hematopoietic stem cells (HSCs), thus increasing their numbers while maintaining their stem cell properties. MenSCs have been tested only in three different animal disease models, exhibiting regenerative properties under different physiological and pathological conditions; however, further investigation is required to substantiate their real therapeutic potential [3-6,12]. From a clinical translational point of view, these stem cells appear to have the robustness to be widely expanded for clinical applications [13] similar to those of BM-MSCs. Indeed, several pilot trials using MenSCs are in the 'recruitment' phase (source: clinicaltrial.gov): Subsidiaries of Medistem (AZ, USA) have already allogenically injected 20 patients with heart disease. Furthermore, S-Evans Biosciences Co., Ltd. (China) initiated the recruitment phase of donors for a clinical study to test the effect of autologous MenSCs in liver cirrhosis, acute lung injury and for the treatment of type 1 diabetes in men and women, in this case with allogeneic MenSCs. These trials attest to the fast path stem cell-based therapies are taking during this decade, from the date of their discoveries to the time they reach the clinic.

Aiming at more clarity in this regard, we have characterized several MenSCs derived from multiple donors using a large battery of markers, aiming not only for typical mesenchymal and embryonic markers but also for endothelial and epithelial lineage markers, since these cell types might represent a source of contamination in MenSCs culture. In addition, we have addressed a range of functional properties that contribute to understanding the regenerative potential that could be expected with preclinical or clinical application of MenSCs. These quality-control parameters are essential when comparing similar cells from different sources. We demonstrate that MenSCs have a considerable potential for self-renewal and stable proliferation in vitro during a long culture time and a significantly higher migration capacity than BM-MSCs, suggesting they might exhibit several unexpected therapeutic capacities. We also demonstrate that MenSCs secrete higher amounts of angiogenic factors than BM-MSCs, resulting in a higher angiogenic potential both in vitro and in vivo. Finally, we demonstrate for the first time that MenSCs possess enhanced feeder properties evidenced by their support of HSCs proliferation. The panel of characteristics presented in this article contributes in orienting their clinical use towards the most appropriate applications.

\section{Methods}

\section{Isolation and culture of cells}

All the cells used in this study were harvested with the informed consent of the donor as approved by an Institutional Review Board of Universidad de los Andes and the ethical scientific committee of Clinica Santa Maria and the 'Servicio de Salud Metropolitano Oriente.' Menstrual blood-derived stem cells (MenSCs) were collected from five healthy donors ranging in age from 18 to 39 years. Samples were collected in a menstrual silicone cup (Mialuna ${ }^{\circ}$, Santiago, Chile) during the earliest days of a menstrual cycle. Menstrual blood samples were transferred into a $50 \mathrm{ml}$ tube with $10 \mathrm{ml}$ phosphate buffered saline (PBS) containing $0.25 \mathrm{mg} / \mathrm{ml}$ amphotericin B, penicillin $100 \mathrm{IU}$, streptomycin $100 \mathrm{mg} / \mathrm{ml}$ and 
$2 \mathrm{mM}$ ethylenediaminetetraacetic acid (EDTA) (all from Gibco, Paisley, UK). Menstrual blood mononuclear cells were separated by Ficoll-Paque Plus (GE Healthcare, Amersham, UK) $(1.077 \mathrm{~g} / \mathrm{ml})$ density gradient according to the manufacturer's instructions and washed in PBS. Cells were subsequently cultured in a T25 flask (Falcon', Becton Dickinson, USA) containing (Dulbecco's) modified Eagle's medium (DMEM) high glucose (Gibco) supplemented with $1 \%$ penicillin/streptomycin $(\mathrm{P} / \mathrm{S}), 1 \%$ amphotericin $\mathrm{B}$, $1 \%$ glutamine (Gibco) and 15\% fetal bovine serum (FBS) (Lonza, Walkersville, MD USA) at $37^{\circ} \mathrm{C}, 5 \% \mathrm{CO} 2$ in order to obtain adherent cells. Media were changed the next day to wash non adherent cells. Cells were seeded again as follows: adherent cells were detached using $0.05 \%$ trypsinEDTA (Gibco), counted and sub-cultured. Bone marrow derived mesenchymal stem cells (BM-MSCs) were collected by bone marrow (BM) aspirates from three hip surgery patients (60 to 72 years old). Additional BM aspirates were obtained by iliac crest puncture of a 13 year old patient. BM-MSCs were grown under the same conditions as MenSCs. All the experiments were performed using MenSCs and BM-MSCs from three different donors at early passages (P) P2 to P6. All cells were routinely tested for mycoplasma. HSCs were collected from fresh umbilical cord blood and mononuclear cells were isolated after centrifugation on Ficoll-Paque Plus. HSCs were immunomagnetically isolated using the EasySep ${ }^{\text {ma }}$ Human Cord Blood CD34 Positive Selection Kit (Stem Cell Technologies, Vancouver, Canada) according to the manufacturer's instructions.

\section{Tri-lineage differentiation}

For adipogenesis, cells were seeded at 30,000 cells/well in a four-well plate, in DMEM media supplemented with $10 \%$ FBS, $\mathrm{P} / \mathrm{S}$ and glutamine. When cells reached sub-confluence, the medium was changed to (D)MEM $10 \% \mathrm{FBS}, \mathrm{P} / \mathrm{S}$ supplemented with $1 \mathrm{umol} / \mathrm{L}$ dexamethasone, $60 \mathrm{umol} / \mathrm{L}$ indomethacin and 50 umol/L IBMX for 14 days. Adipocyte differentiation was quantified by measuring the expression levels of peroxysome proliferator-activated receptor (PPAR)$\gamma$ by RT-PCR and by the formation of lipid droplets visualized by OIL RED O stain in an inverted light microscope.

For osteogenesis, cells where seeded at 40,000 cells/well in a four-well plate. At confluence, medium was changed to DMEM 10\% FBS, supplemented with $0.1 \mathrm{umol} / \mathrm{L}$ dexamethasone and $50 \mathrm{ug} / \mathrm{mL}$ ascorbic acid in the presence or absence of $3 \mathrm{mM}$ of $\mathrm{Na}_{2} \mathrm{HPO}_{4}$ for matrix mineralization or RT-PCR analysis, respectively, for 21 days. Osteogenesis was quantified by osteocalcin (OC) expression levels by RT-PCR and by Alizarin Red S positive staining.

Chondrogenesis was performed by culturing either BMMSCs or MenSCs $\left(2.5 \times 10^{5}\right.$ cells $)$ in micropellet in the presence of DMEM supplemented with $0.1 \mathrm{uM}$ dexamethasone, $0.17 \mathrm{mM}$ ascorbic acid, $1 \%$ insulin-transferrin-selenic acid
(ITS) and $10 \mathrm{ng} / \mathrm{ml}$ transforming growth factor b3 (TGFb3) and cultured for 21 days. The chondrogenesis potential was assessed by aggrecan and collagen II RNA expression levels and by positive staining for Safranin O.

\section{RNA expression}

Total RNAs were extracted using the RNeasy kit (Qiagen, Courtaboeuf, France). RNA (0.5 $\mu \mathrm{g})$ was reverse-transcribed using the M-MLV enzyme (Fisher Scientific, Illkirch, France) and $25 \mathrm{ng}$ of cDNA were amplified using a LightCycler 480 system (Roche Diagnostics, Meylan, France) using the following forward and reverse primers: Aggrecanforward primer (F): 5' -TCGAGGACAGCGAGGCC-3', Aggregan-reverse primer (R): 5' - TCGAGGGTGTAGC GTGTAGAGA-3'; Collagen IIA-F: 5'-ACGTGAAAGACTGCCTCAGC-3', Collagen IIA-R: 5' - AGGAGG TCTTTGGGTCCTA-3'; RSP9-F: 5'-ATGAAGGACG GGATGTTCAC-3', RSP9-R: 5' - GATTACATCCTGGG CCTGAA-3'; PPAR $\gamma$-F: 5' -CCAGAAAGCGATTCCT TCAC-3', PPAR $\gamma$-R: 5' - TGCAACCACTGGATCTGT TC-3'; Osteocalcin-F: 5'-GGCGCTACCTGTATCAAT GG-3', Osteocalcin-R: 5' - TCAGCCAACTCGTCACA GTC-3'. All values were normalized to either Bglobulin or RPS9 housekeeping gene and expressed as relative expression or fold change using the respective formulae $2^{-\Delta \mathrm{CT}}$ or $2^{-\Delta \Delta \mathrm{Ct}}$.

\section{Proliferation assay}

MenSCs and BM-MSCs were cultured at 1,000 cells $/ \mathrm{cm}^{2}$ in 24-well plates (Falcon ${ }^{\circ}$, Becton Dickinson) in complete DMEM media. Cell proliferation and viability were determined at days 3, 6 and 9 by measuring cellular mitochondrial dehydrogenase using the Quick Cell Proliferation Assay Kit II (BioVision, Milpitas, CA USA) and by spectrophotometric quantification (absorbance, $450 \mathrm{~nm}$ ) according to the manufacturer's instructions. Three independent experiments were performed in triplicate. Student's $t$-test was used to calculate statistical difference. A $P$ value $\leq 0.05$ was considered to be significant.

\section{In vitro scratch assay}

Cell migration capacity was evaluated in a scratch assay, where cells were grown in six-well plates (Falcon', Becton Dickinson) to full confluence. A straight scratch of the cell monolayer was performed with a $10 \mu \mathrm{l}$ pipet tip. Cells were washed with PBS to remove debris and incubated with DMEM 2\% FBS for 24 hours. Images were acquired for each sample under a phase-contrast microscope at defined time frames to monitor cell migration into the ruptured area. Migration abilities were quantified by the number of migrated cells inside the scratch area using ImageJ analysis software. The experiment was performed in triplicate. Student's $t$-test was used to calculate statistical difference. A $P$ value $\leq 0.05$ was considered to be statistically significant. 


\section{Colony forming unit assay}

To quantify the frequency of stromal progenitors, mononuclear cells obtained after ficoll centrifugation of the menstrual blood were resuspended in DMEM and plated at a density of 100,1,000,10,000 and 100,000 nucleated cells $/ \mathrm{cm}^{2}$. The medium was changed the next day to wash non adherent cells. The frequency of progenitors was calculated following the extreme limiting dilution analysis (ELDA) method for comparing depleted and enriched populations in stem cells [14]. To quantify functional mesenchymal stem cells, MenSCs and BM-MSCs were evaluated for frequency of fibroblast colony-forming units (CFU-F). CFU-F between passage (P) 3 and P 6 were evaluated in a serial dilution assay, where 25 to 250 cells per well were seeded in a six-well plate (Falcon ${ }^{\circ}$, Becton Dickinson). After nine to twelve days, cells were stained with $0.5 \%$ crystal violet (Sigma-Aldrich, St.Louis, MO, USA) in $10 \%$ methanol for 20 minutes. After several washes, colonies formed by more than 50 fibroblast-like cells were counted under a light microscope at low magnification. Results were expressed as CFU/number of cells plated. The Student's $t$-test was used to detect statistical differences at a $P$ value of $P \leq 0.05$.

\section{Immunophenotypic characterization of MenSCs}

For cell surface antigen analysis, cells were harvested, washed with cytometer buffer (PBS $+0.2 \%$ BSA $+0.01 \%$ sodium azide (all from Sigma-Aldrich) and incubated with the specific labelled antibodies in cytometer buffer for 20 minutes at $4^{\circ} \mathrm{C}$. Antibodies for human cell surface antigens CD14, CD44, CD90, CD271, CD105, CD73, CD117, CD45, CD34, HLA-ABC, HLA-DR, CD146, EPCAM, CD31, TRA-1-60, CD49a, SSEA3, SSEA4, CD133 and CD3 were purchased from BD Pharmingen ${ }^{\mathrm{Tm}}$ (BD Biosciences, San Jose, CA, USA), R\&D Systems (Minneapolis, MN, USA) and Biolegend (San Diego, CA, USA). In all experiments, matching isotype antibodies were used as negative controls. In addition, LIVE/DEAD ${ }^{\circ}$ Fixable dead cell stain kit (Invitrogen, CA, USA) was used to determine the viability of cells by flow cytometry according to the manufacturer's protocol. Data (5,000 events) were collected using a fluorescence-activated cell sorting (FACS) Canto II Flow cytometer (BD Biosciences) and analyzed on Flowjo analysis software.

\section{Angiogenesis assay}

Tube formation in Matrigel was used as a functional assay to evaluate the angiogenic properties of MenSCs. Human umbilical vein endothelial cells (HUVEC) and MSCs were plated at 3,000 cells $/ \mathrm{cm}^{2}$ and grown until confluence. Cells were incubated for 24 hours in DMEM 2\% FBS and then exposed to normoxic and hypoxic conditions $\left(1 \% \mathrm{O}_{2}\right)$ for 48 hours. Then, conditioned medium (CM) was collected, centrifuged at $500 \mathrm{~g}$ for five minutes, and stored in aliquots at $-80^{\circ} \mathrm{C}$. Cells were trypsinized and resuspended in the $\mathrm{CM}$ or in endothelial growth medium (Lonza). Cells were then seeded in triplicate in four-well plates (Falcon', Becton Dickinson) pre-coated with $200 \mu \mathrm{l}$ growth factor reduce Matrigel (BD Biosciences, USA) at a density of $6 \times$ $10^{4}$ cells/well according to the manufacturer's instructions. After six hours, gels were examined by phase-contrast and three images per well were capture using an Olympus URFL-T camera. Covered area, total loops and total length of tube were analyzed using the WinTube software (Wimasis, Munich, Germany). The experiment was performed in triplicate. The Student's $t$-test was used to calculate statistical difference. A $P$ value of $P \leq 0.05$ was considered to be statistically significant.

\section{Quantification of secreted factors by ELISA}

MSCs were grown in a six-well plate $\left(5 \times 10^{5}\right.$ cells/well $)$ and incubated overnight in complete media. MSCs were washed with PBS and cultured for 72 hours with serumfree DMEM in normoxic and hypoxic $\left(1 \% \mathrm{O}_{2}\right.$, $)$ conditions. Conditioned medium was collected and centrifuged at $500 \mathrm{~g}$ for five minutes to remove debris. VEGF and basic fibroblast growth factor (bFGF) levels were detected using VEGF and bFGF duo set ELISA (R\&D Systems) according to the manufacturer's protocol. The Student's t-test was used to calculate statistical difference. A $P$ value of $P \leq 0.05$ was considered to be statistically significant.

\section{In vitro expansion of cord blood CD34 + CD133+ hematopoietic stem cells}

CD34+ hematopoietic progenitor cells (HPCs) were purified as described previously [11]. The percentage of CD34+ CD133+ HSCs upon enrichment was determined by flow cytometry $(76.8 \% \pm 7.99 ; n=5)$. Confluent layers of MenSCs and BM-MSCs were irradiated (15Gy) at the Chilean Commission for Nuclear Energy Facilities. CD34+ hematopoietic progenitor cells were then co-cultured directly in the presence of the feeder layer (contact conditions) or in their absence (HSCs alone) or separated from it by a $0.4 \mu \mathrm{m}$ microporous membrane (Transwell, BD Bioscience) (noncontact conditions) using StemSpan (StemCell Technologies, Vancouver, BC, Canada) supplemented with human growth factors: $10 \mathrm{ng} / \mathrm{ml}$ fibroblast growth factor 1 (FGF-1), $10 \mathrm{ng} / \mathrm{ml}$ stem cell factor (SCF), $20 \mathrm{ng} / \mathrm{ml}$ thrombopoietin (TPO), $100 \mathrm{ng} / \mathrm{ml}$ insulin-like growth factor-binding protein 2 (IGFBP2) (all from R\&D Systems, Minneapolis, USA), $100 \mathrm{ug} / \mathrm{ml}$ of heparin and P/ S (Gibco) as previously described [11]. Briefly, CD34+ HPCs were plated directly on these feeder layers or in transwells above the feeder layer or without stroma in a 1:4 to 1:5 ratio in growth factor-supplemented StemSpan in 24-well plates (Falcon', Becton Dickinson) at $500 \mu \mathrm{l} /$ well. Half of the medium was changed every day and was replaced with an equal volume of fresh media. 


\section{Proliferative and phenotypic analysis of CD34 + CD133+ hematopoietic stem cells}

All the expanded cord blood cells were harvested after three, five and seven days by vigorous pipetting, washed in PBS, and the total number of live cells determined using trypan blue staining. The fold increase in CD34 + CD133+ HSCs, CD34+ HPCs and total cells was calculated by dividing the number of cells at each day by the initial number of cells plated at day 1 . In addition, cells were stained with CD3-APC, CD45-FITC, CD34-PECy7, CD133-PE, and LIVE/DEAD ${ }^{\circ}$ Fixable dead cell stain. Expression of surface markers was then analyzed by flow cytometry. The absolute number of expanded CD34+ or CD34 + CD133+ cells was obtained by multiplying the percentage of CD34+ or CD34 + CD133+ cells with the total number of cells counted in the same culture well. Three independent experiments were performed each in triplicate. Student's $t$-test was used to calculate statistical difference. A $P$ value of $P \leq 0.05$ was considered to be statistically significant.

\section{Hematopoietic colony assay}

Expanded CD34 + CD133+ hematopoietic stem cells were resuspended in Iscove's MDM plus 2\% FBS and plated on methylcellulose-based media (Methocult ${ }^{\mathrm{tm}}$ Optimum, StemCell Technologies) according to the manufacturer's instruction. Cells were plated, in duplicate, at an equivalent final concentration to initial 50 cord blood CD34+CD133+ hematopoietic stem cells and incubated for 14 days at $37^{\circ} \mathrm{C}$ with $5 \% \mathrm{CO}_{2}$ and $\geq 95 \%$ humidity. Burst-forming unit-erythroid (BFU-E), colonyforming unit granulocyte and macrophage (CFU-GM) and colony-forming unit-granulocyte, erythroid, macrophage and megakaryocyte (CFU-GEMM) were classified and counted according to morphological criteria under microscopy. Colonies with more than 50 cells were counted as positive. Student's $t$-test was used to calculate statistical difference. A $P$ value of $P \leq 0.05$ was considered to be statistically significant.

\section{In vivo animal models}

All in vivo studies were performed at the animal facility of the Universidad de los Andes (Santiago, Chile) and received approval by the 'Universidad de Los Andes ethical committee for animal experimentation.' In order to evaluate the angiogenic potential of MenSCs, matrigel plug assay was performed in eight-week-old NSG mice (NOD.Cg-Prkdcscid Il2rgtm1Wjl/SzJ, Jackson Laboratories, Bar Harbor, ME, USA). For the matrigel plug assay, mice were randomly divided into four groups ( $n=6 / 8$ plugs per group): DMEM (as negative control), HUVEC, BM-MSCs and MenSCs. Growth factor-reduced matrigel (BD Bioscience) was mixed with (D)MEM alone (supplemented with $50 \mathrm{ng} / \mathrm{ml}$ VEGF) or $3 \times 10^{6}$ cells (resuspended in DMEM supplemented with $50 \mathrm{ng} / \mathrm{ml}$ VEGF), and subcutaneously injected into both flanks of mice. Fourteen days later, matrigel plugs were harvested and processed for hemoglobin quantification. In brief, the matrigel plugs were homogenized in $250 \mu \mathrm{l}$ Brij-35 solution (Sigma), cleared by centrifugation at 5,000 $\mathrm{g}$ for five minutes and the supernatants were collected to measure hemoglobin content with Drabkin's solution (Sigma) according to the manufacturer's instruction. Student's t-test was used to calculate statistical difference. A $P$ value of $P \leq 0.05$ was considered to be statistically significant.

\section{Results}

\section{MenSCs exhibit higher proliferation rate, CFU-frequency} and migration capacity

Multiple in vitro assays were performed to compare distinct properties of MSCs isolated from the menstrual fluid with the gold-standard BM-MSCs. Following cell isolation, MenSCs exhibited spindle fibroblast-like morphology in culture and expressed mesenchymal stem cells surface markers, such as CD105, CD90, CD73, and CD44, in the absence of hematopoietic cell surface markers including CD34, CD45, and CD14. Furthermore, MenSCs lacked the expression of HLA-DR, CD271, CD117, and the endothelial and epithelial surface marker CD31 and EPCAM, respectively. Minimal levels of expression were detected for CD146. While both MSCs sources express HLA-ABC, MenSCs showed higher mean fluorescence intensity. The only prominent immuno-phenotypic difference noted between the two cell sources was the high expression of the $\alpha 1 \beta 1$ integrin (CD49a). In addition, MenSCs were negative for the pluripotential surface markers hTRA-1-60, SSEA3 and SSEA4 (Figure 1A). The phenotype identified was stable throughout the subculture period from early passages (P3 to P6) to late passages (P12 to P14) (see Additional file 1: Figure S1).

To evaluate the differentiation capacity of MenSCs into mesodermal tissues, we induced BM-MSCs and MenSCs to differentiate into adipocytes, osteoblasts and chondrocytes. Our results showed that both BM-MSCs and MenSCs were able to differentiate into all three lineages (Figure 1B). However, the quantification of $\mathrm{PPR} \gamma$ levels and oil red $\mathrm{O}$ staining revealed that BM-MSCs exhibit significantly higher adipogenic differentiation potential. Nevertheless, no difference was noted when evaluating their osteogenic and chondrogenic differentiation potential by OC and Alizarin red staining and aggrecan, Col2A expression levels and Safranin O staining, respectively.

In accordance with other reports, we observed that MenSCs exhibit a higher proliferation rate [5,7]. The proliferation levels evaluated at culture days 3,6 and 9 by cellular mitochondrial dehydrogenase measurement, showed a significant 2.84 fold increase $(P \leq 0.05)$ at day 6 and 4.97 fold increase at day $9(P \leq 0.01)$ of the MenSCs in comparison with the BM-MSCs (Figure 2A). Moreover, the proliferation levels remained unchanged when the cells were 


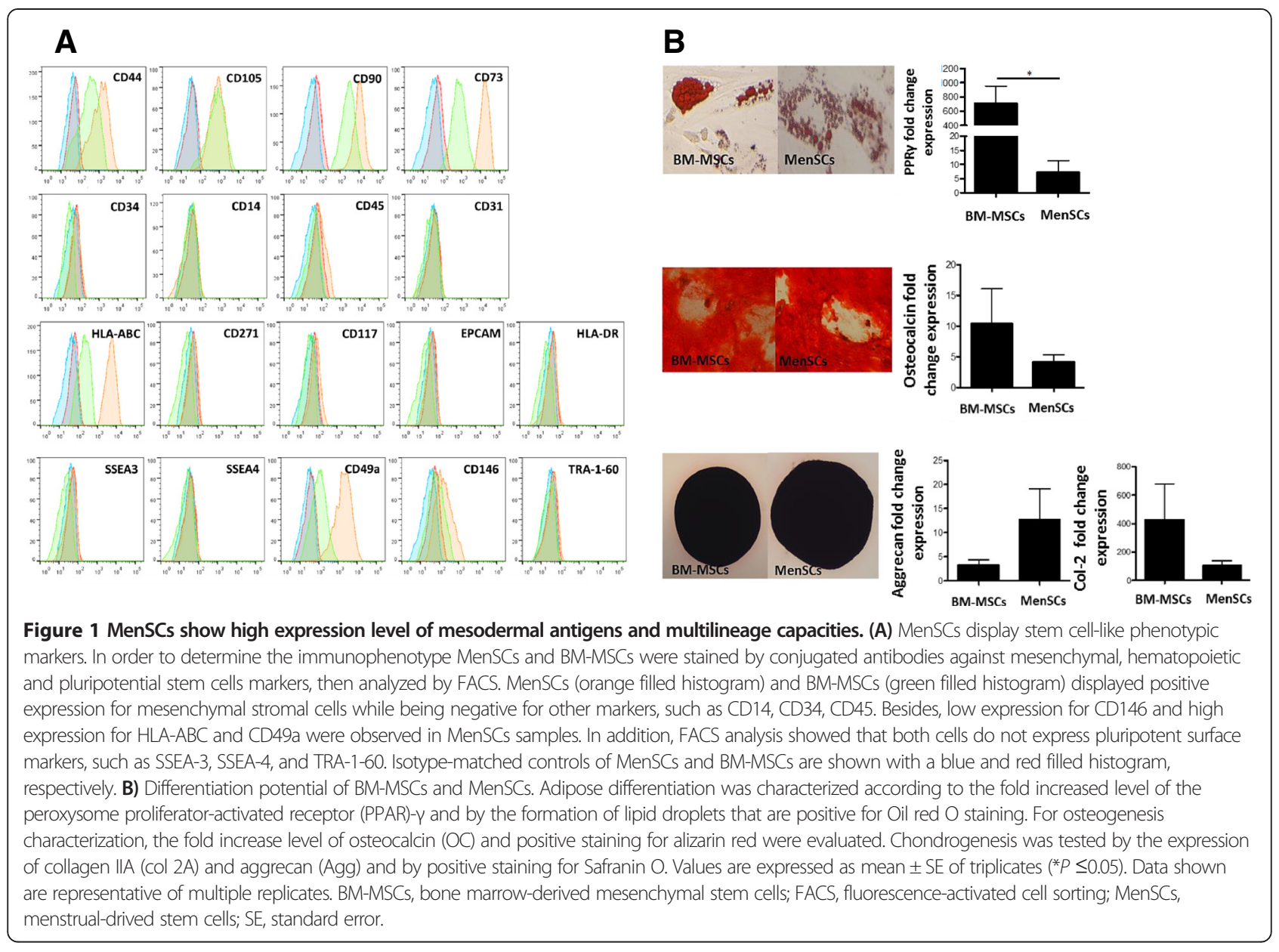

cultured for a longer period (P12 to P14) (see Additional file 2: Figure S2).

To evaluate the initial frequency of mesenchymal progenitors in menstrual fluids, the CFU-F assay was performed immediately after ficoll isolation from freshly obtained samples. The frequency of CFU-F was calculated based on the culture of cells isolated from 10 independent samples and serially diluted at numbers ranging from 100 to $10^{5}$. The value of $1 /$ (CFU frequency) calculated from the limiting dilution analysis was 1 in 10,104 with 95\% confidence interval (CI) of $1 / 4,787$ to $1 / 21,325$ (see Additional file 3: Figure S3). Additionally, we performed a CFU-F assay after cell expansion at early passages (P3 to P6) to compare their CFU potential with BM-MSCs. After serial dilution of a normalized number of total cells from both sources, MenSCs generated a significantly higher number of CFUs in comparison to BM-MSCs, with an average 3.5-fold increase in almost all dilutions examined (Figure 2B). The morphology of the colonies generated from the MenSCs was more dense and larger in size and cell number as shown in Figure 2C. In order to assess the stability of their CFU potential, MenSCs were also seeded at a later passage (P12 to P14), with no significant changes noted between early and late passages (see
Additional file 4: Figure S4). Furthermore, it is important to note that similar to a previous report regarding donor-todonor differences for BM-MSCs, MenSCs also presented variability in their properties that were donor dependent [21]. This was evidenced by the comparison of ten samples from different donors in their CFU-potential and absolute progenitor numbers (see Additional file 5: Figure S5).

A scratch wound assay was then performed to examine the migration ability of MenSCs in vitro during wound closure. Images taken at different time-lapses post-scratch, showed higher migration potential of MenSCs with respect to BM-MSCs, where complete wound closure was observed within 24 hours. In contrast, BM-MSCs showed only a partial closure after 24 hours (Figure 2D). The number of migrated cells was quantified using digital image analysis software and resulted in a statistically significant higher migratory capacity of MenSCs at 12 hours $(P \leq 0.01)$ and 24 hours post-scratch $(P \leq 0.05)$ (Figure $2 \mathrm{E})$.

\section{MenSCs show higher angiogenic effect on endothelial cells both in vitro and in vivo}

MSCs share different histological properties that are coherent with endothelial cells enabling them to contribute 


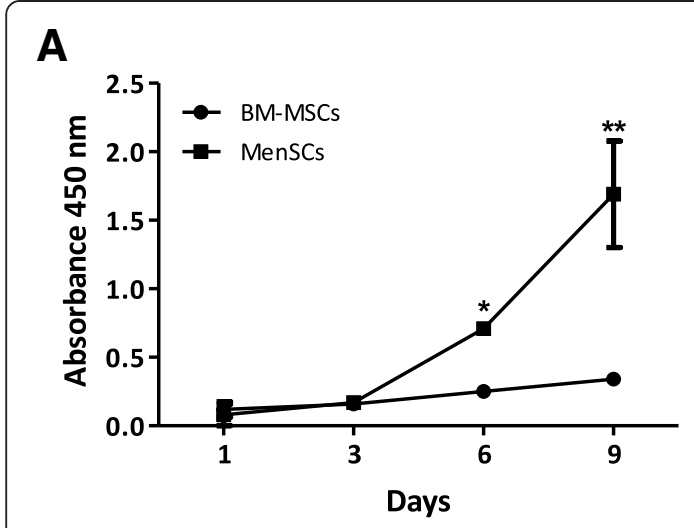

D

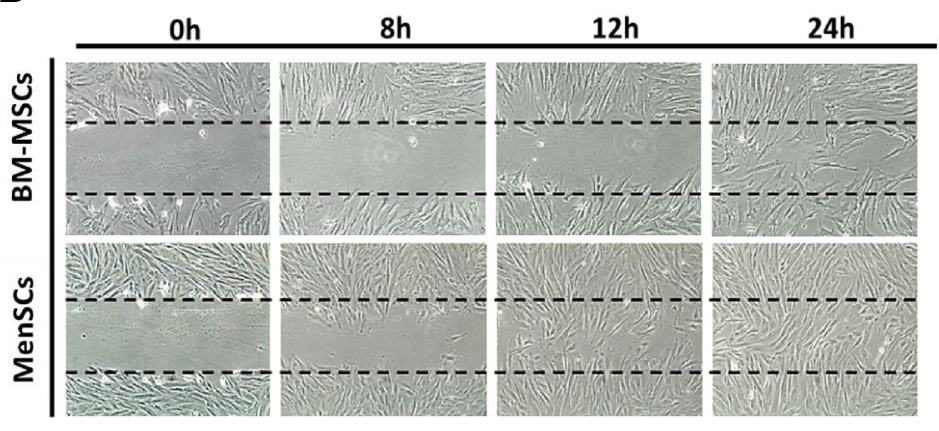

B

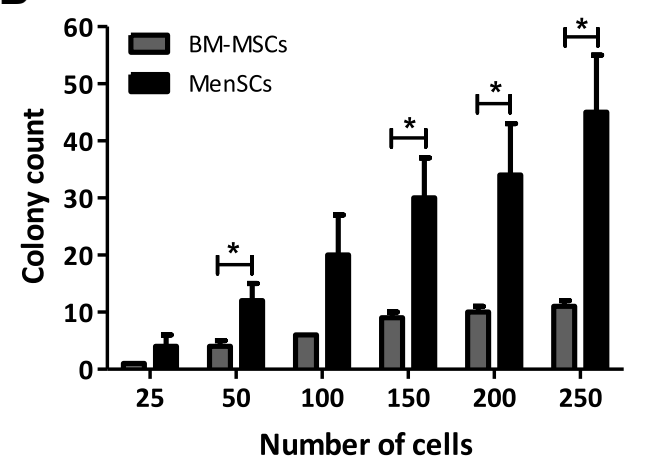

C

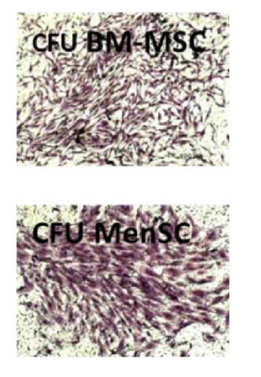

E

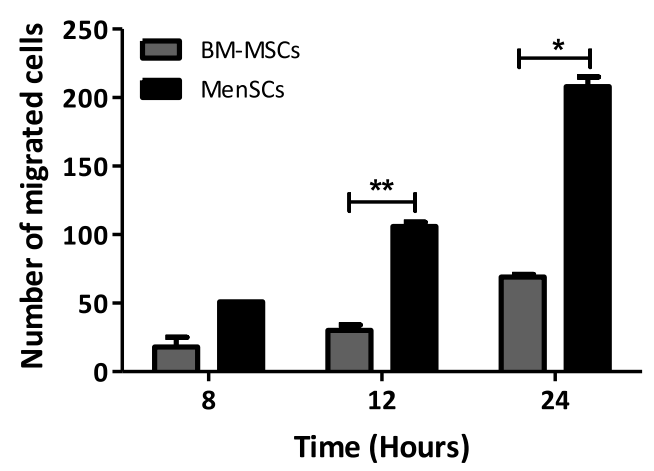

Figure 2 MenSCs display high proliferation potential, CFU-F capacity, and migration ability. (A) MenSCS show high proliferation. Proliferation was evaluated using cellular mitochondrial dehydrogenase quantification at different time points. MenSCs showed significantly higher proliferation kinetics with respect to BM-MSCS. (B) CFU-F capacity. Serial dilutions of a defined number of cells were cultured and the potential to form CFU was evaluated. MenSCS significantly generated more CFU than BM-MSCs. (C) CFU-F morphology of mesenchymal stem cells. At 21 days of culture, the CFU were stained with crystal violet to visualize the colonies generated. (D-E) Scratch assay. Confluent monolayers of MenSCs and BM-MSCs were mechanically disrupted with a sterile p10 pipet tip. Images were acquired under a phase-contrast microscope. (D) Panels show representative images of BM-MSCs and MenSCs migration post-scratch assay obtained at different time points (magnification 10x). (E) Graph represents statistical analysis of scratch assay. Values are expressed as mean \pm SE (*P $\leq 0.05$; **P $\leq 0.01$ ). Data shown are representative of multiple replicates. BM-MSCS, bone marrow-derived mesenchymal stem cells; CFU-F, fibroblast colony-forming unit; MenSCs, menstrual-drived stem cells; SE, standard error.

directly to angiogenesis. They are also capable of participating through the regulation of their secretion of proangiogenic factors under an atmosphere of low oxygen that closely approximates the ischemic microenvironment. Hence, the ability of the MenSCs to form a tubule network in semi-solid medium was first tested in an in vitro angiogenesis assay. MenSCs and BM-MSCs were resuspended in basal medium and seeded on a threedimensional matrigel culture system. Tube formation starts to appear at six hours post-culture initiation; however, the difference between BM-MSCs and MenSCs was not significant at that time point (data not shown). In a similar assay, angiogenic factors released by MenSCs and BM-MSCs in the respective conditioned media (CM) were evaluated using HUVEC. In order to mimic an ischemic condition in vitro, the effect of CM collected under different oxygen conditions (normoxia versus hypoxia) was evaluated on the capillary-like tube formation of HUVEC (Figure 3). Image analysis of the tube formation showed a more extensive network of capillary-like structures in response to MenSCs CM as compared to BM-MSCs CM or unconditioned control medium (Figure 3A). MenSCs CM cultured under hypoxia showed a significant stimulation in capillary-like structure formation than the medium from cells cultured under normoxic conditions (Figure 3B-D). However, BM-MSCs CM cultured under the different $\mathrm{O}_{2}$ levels had no significant difference in tube formation. Under normoxic condition, MenSCs CM showed a higher percentage of covered area $(26.48 \pm 1.19 \%)$ versus BM-MSCs CM $(15.68 \pm 0.24 \%, P \leq 0.0001)$, higher loop numbers $(12.09 \pm 1.19)$ versus BM-MSCs CM $(3.001 \pm 0.47$, $P \leq 0.0001)$ and also longer tube length $(20,718.64 \pm$ 531.54 pixels ) versus BM-MSCs CM (16,476 \pm 454.14 pixels, $P \leq 0.0001)$. In accordance with the data presented above, MenSCs CM from hypoxic cultures induced significantly more capillary-like formation than the normoxic condition in all the parameters we evaluated: percentage 


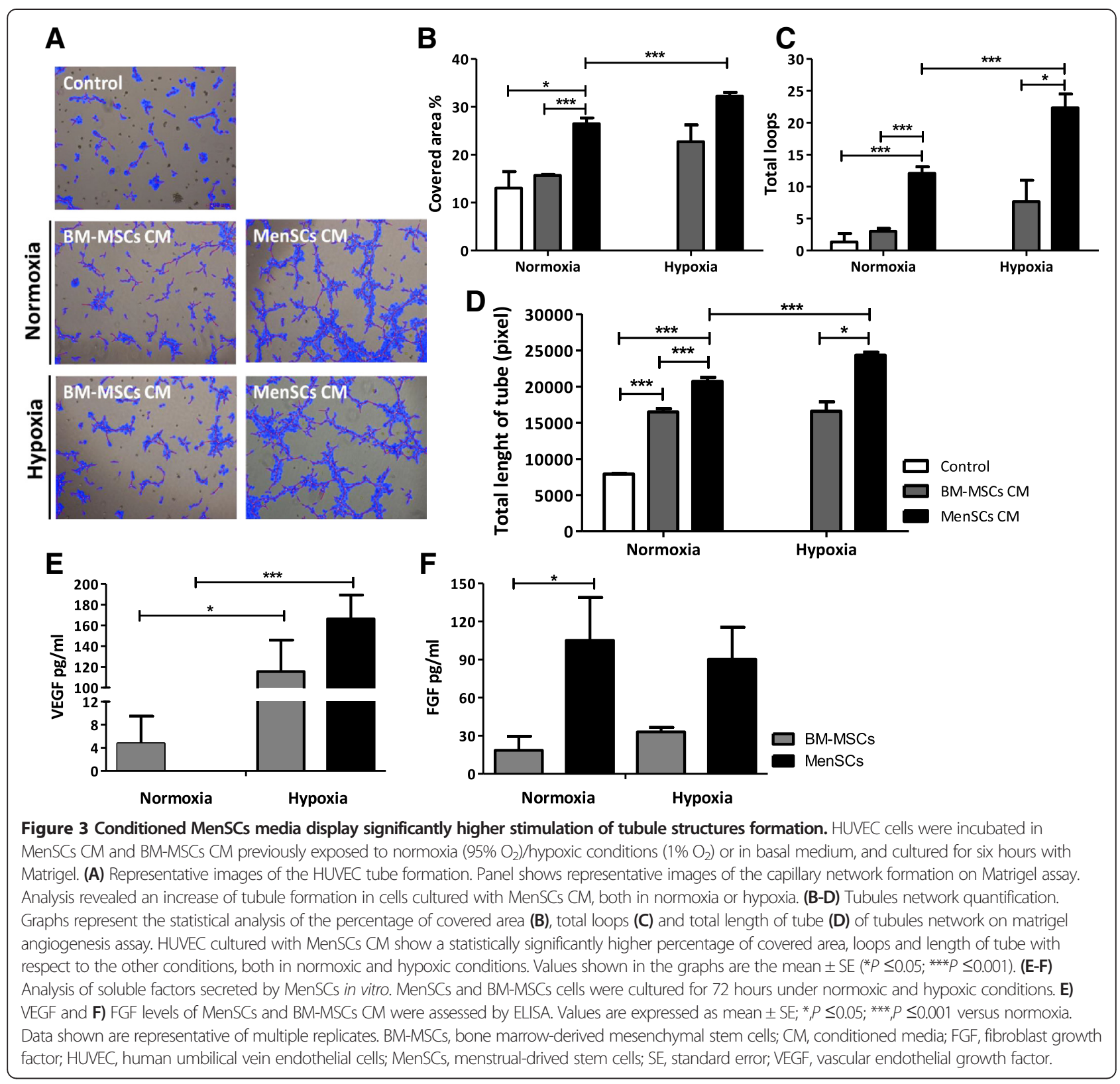

of covered area $(P \leq 0.001)$, total loop number $(P \leq 0.001)$ and total tube length $(P \leq 0.0001)$. Moreover, the difference in all the parameters mentioned above remained significantly higher in the MenSCs CM conditions. The secreted levels of the angiogenic factors VEGF and bFGF were measured in order to explain the difference in the outcome observed between the two different sources and under different oxygen conditions. Under hypoxic conditions, an important increase of the VEGF secretion level was noted in both CM (24 and 174 folds for BM-MSC and MenSCs, respectively), in comparison with normoxic conditions (Figure 3E). On the other hand, MenSCs CM showed significantly higher bFGF levels in comparison with
BM-MSCs CM, under both oxygen conditions (Figure 3F).

To evaluate the angiogenic potential of MenSCs in vivo, matrigel plug assays were performed in immunocompromised mice. MSCs were mixed with the matrigel and transplanted subcutaneously in mice. At 14 days post-transplantation, plugs were harvested for macroscopic analysis and hemoglobin quantification (Figure 4A). A larger number of blood vessels surrounding the inserted plug was observed with MenSCs; this was correlated with a significant increase in hemoglobin content $(212.54 \pm 8.72 \mathrm{mg} / \mathrm{dl})$ compared to BM-MSCs $(26.5 \pm 8.67 \mathrm{mg} / \mathrm{dl})$ or DMEM $(14.53 \pm 4.95 \mathrm{mg} / \mathrm{dl})$ 
A

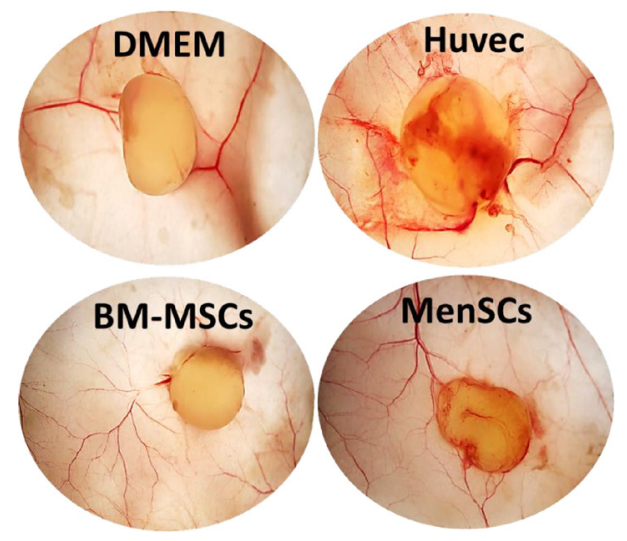

B

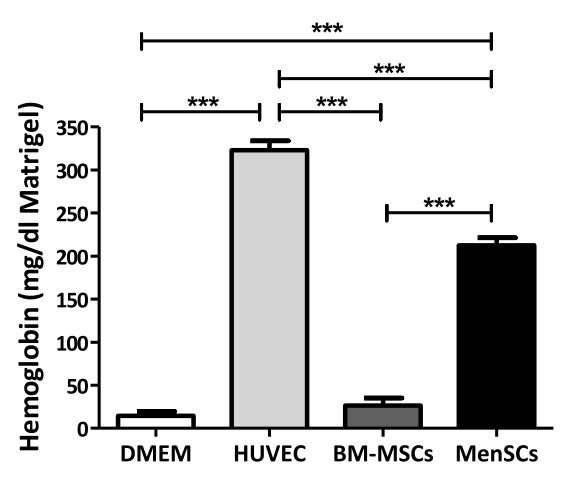

Figure 4 MenSCs promote angiogenesis in vivo. (A) Matrigel was mixed with cells $\left(3 \times 10^{6}\right.$ cells/plug) or DMEM alone and subcutaneously injected into mice. After 14 days, the matrigel plugs were harvested and photographed. (B) MenSCs have a high hemoglobin content. The hemoglobin content of the plugs from the different groups was measured using Drabkin's method. The graph represents statistical analysis of the hemoglobin concentration in the matrigel plugs. Values are expressed as mean \pm SE (**P $\leq 0.001)$. MenSC, menstrual-derived stem cells; SE, standard error.

$(P \leq 0.001)$, suggesting that pro-angiogenic effects of MenSCs are also seen in vivo (Figure 4B).

\section{MenSCs strongly support the ex vivo proliferation of hematopoietic stem cells in a cell contact dependent manner}

MSCs present stromal support properties that can positively impact the expansion and preservation of the 'stemness' potential of HSCs/HPCs. In the interest of determining whether the MenSCs possess this property, human umbilical cord blood CD34 + CD133+ cells were isolated and expanded under different culture conditions. First, HSCs were grown directly on confluent MenSCs- or BM-MSCs-feeder layers allowing direct cell-to-cell contact (Figure 5E).

Both co-culture sources were compared to the condition where HSCs were expanded in the absence of feeder cells. Cocultures were maintained for seven days in serum-free defined media containing heparin, SCF, TPO, FGF-1 and IGFBP-2 as previously described [11]. Starting at a density of approximately $1 \times 10^{4}$ cells/well, the expansion of $\mathrm{CD} 34^{+}$ $\mathrm{CD} 133^{+}$cells (early progenitors), $\mathrm{CD} 34^{+} \mathrm{CD} 133^{-}$(late progenitors) and total expanded cells (CD34 ${ }^{+}$and CD34- cells, presenting differentiated cells) was assessed at different time points of culture (Figure 5). The percentage of expanded hematopoietic progenitors and stem cells was evaluated by flow cytometry, based on the expression of the CD34 and CD133 surface markers at day 1 and 7 of culture (Figure 5D). The proliferation of HSCs in vitro occurs both through selfrenewal and also differentiation toward early and late hematopoietic progenitors [11]. This explains the decrease in the percentage of the CD34 + CD133+ and CD34+ cells during the expansion time. While the percentage of CD34+ was lower in the condition using the MenSCs-feeder layers, the absolute number of CD34+ cells and, consequently, the level of expansion of these hematopoietic progenitors showed a consistent fold increase of $11.09 \pm 1.16$. In comparison, the BM-MSCs-feeder layer condition yielded a fold increase of $4.51 \pm 0.59$ and HSCs alone reached 4.04 \pm 1.58 . At the end of the experiment, a significant expansion of the CD34+ cells was reached under the MenSCs-feeder layer contact condition with respect to the BM-MSCs-feeder layer $(P \leq 0.0001)$ or HSCs alone conditions $(P \leq 0.005)$ : 2.46 - and 2.75 -fold, respectively (Figure $5 \mathrm{~A}$ ). The expansion fold of CD34 + CD133+ hematopoietic stem cells was achieved in the co-culture condition with the MenSCs-feeder layer (3.31 \pm 0.33$)$ in comparison with the BM-MSCs-feeder layer $(1.89 \pm 0.25 ; P \leq 0.01)$ or HSCs alone $(1.78 \pm 0.52 ; P \leq 0.05)$ (Figure 5B). Although, the percentage of CD34 + CD133+ cells on MenSCs was not different, the total number of cells (CD34+ and CD34- cells) obtained was also significantly increased by direct contact with the MenSCs-feeder layer in comparison to both the BM-MSCs-feeder layer and the feeder-free condition support. As expected, total cell numbers were consistently higher with MenSCs-feeder layers compared to BM-MSCs-feeders $(P \leq 0.05)$ or HSCs alone $(P \leq 0.05)$, reaching a $10.35 \pm 0.57$ fold increase (Figure $5 C$ ). The expansion values obtained on the BM-MSCs-feeder layer and HSCs alone were: $3.98 \pm 0.30$ and $3.49 \pm 0.59$, respectively.

Previous reports derived from ex vivo co-culture systems using MSCs as a feeder layer suggest that cell-to-cell contact has a significant impact on cell expansion. In order to determine both the effect of the soluble factors released in the culture and MenSCs contact mediated support, direct and indirect co-cultures between HSCs and the MenSCs-feeder layer (contact condition) were evaluated. To this effect, cocultures were separated by a microporous membrane that 


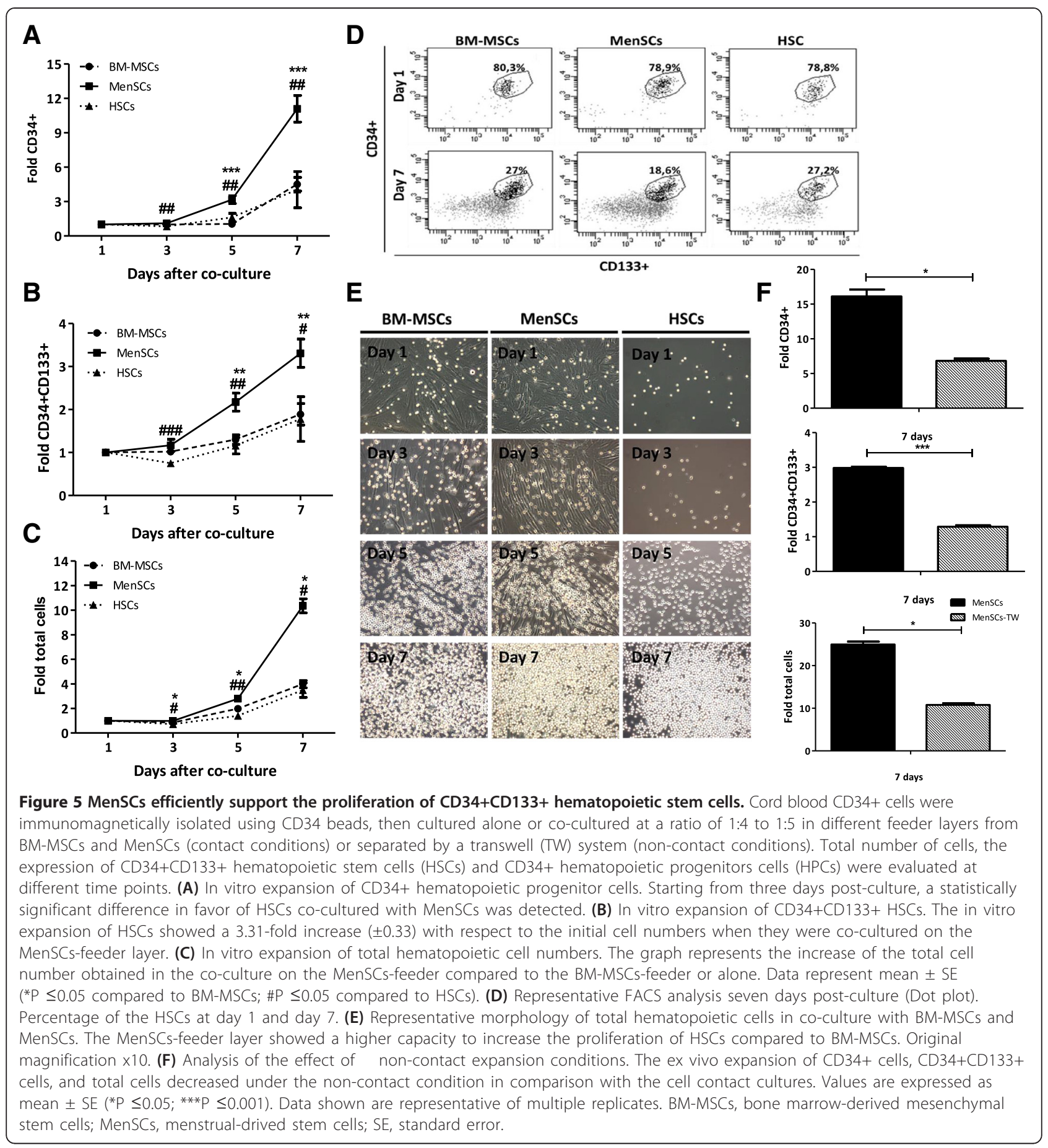

prevented cell contact but allowed exchange of secreted factors. Under these conditions, the expansion of CD34+ cells, CD34+CD133+ cells and total hematopoietic cells was significantly decreased ( 2.3 fold) in the non-contact condition for CD34+ cells and total cells. CD34 + CD133+ cells were more affected by the absence of cell contact where a 1.29 fold-decrease was observed (Figure 5F). These results suggest that $\mathrm{CD} 34+\mathrm{CD} 133+\mathrm{HSC}$ expansion is consistently dependent on direct cell interactions with the stromal cells, more than HPCs or hematopoietic cells.

\section{HSCs expanded on MenSC-feeder layers display higher clonogenic potential}

To assess the changes in the hematopoietic potential of the expanded cells under the different culture conditions, the progeny of $50 \mathrm{CD} 34+\mathrm{CD} 133+$ cells was plated on 
methylcellulose-based media and the number and type of hematopoietic colonies was assessed. Colonies were isolated with the following frequency: for the MenSCs-feeder contact condition the frequency of BFU-E was $30.5 \pm 5.5$; for CFU-GM, $25 \pm 6.0$ and for CFU-GEMM, $302.5 \pm 17.5$; for the BM-MSCs-feeder contact condition the frequency of BFU-E was 24 \pm 5.0 ; and for CFU-GM, $23 \pm 6.0$ and CFU-GEMM, $97 \pm 18$. For the HSC alone condition (absence of stroma) the frequency of BFU-E was $1.0 \pm 1.0$; for CFU-GM, $4 \pm 4$ and for CFU-GEMM it was $206.5 \pm 22.5$. As shown in Figure 6A, significant differences $(P \leq 0.01)$ were observed in the frequency of the most primitive colonies (CFU-GEMM) originated with MenSCs-feeder support as compared to BM-MSCs-feeders, while no difference was detected in BFU-E and CFU-GM colonies under our three different conditions. Representative images of each type of colony derived from hematopoietic progenitors are shown in Figure 6B.

\section{Discussion}

Menstrual fluids are emerging as an alternative source for the isolation of MSCs, devoid of ethical dilemmas or medical complications for cell harvesting. A major drawback of a single sample harvesting, such as placental and bone marrow sources, is the limited number of culture passages before the cells lose the stem potential deemed crucial for clinical efficacy. The next most important practical feature of MenSCs, besides their accessibility and non-invasive isolation procedure, is the possibility of periodic collections from the same donor. Repetitive collection can ensure higher therapeutic doses of low passaged MSCs from the same genetic background. However, this sole advantage is not sufficient to position menstrual blood as a prominent source for cell therapy. It is essential to contrast these advantages with a broader characterization of the functional properties that might underlie specific therapeutic uses. Multiple efforts have focused on identifying and comparing the cellular and molecular aspects of cell morphology, surface markers, and differentiation capacity of stem cells from the various available sources. Within that scope, we have consequently focused our investigation on looking at different parameters including proliferation and migration rates, and also differentiation, angiogenic and cell feeder potential. For the relevance of the study, all the evaluated measurements were compared head-to-head with bone marrow derived cells, the most broadly used source for MSCs. Both cell sources exhibited MSC features as defined by the International Society for Cellular Therapy (ISCT) minimum criteria [15] including spindle shape, multi-lineage differentiation, and surface marker expression. The phenotypic analysis of MenSCs revealed a profile largely similar to the BM-MSCs with the exception of a higher expression of CD49a (alpha1-integrin subunit). CD49a is an adhesion molecule involved in the regulation of leukocyte migration, proliferation, and cytokine production [16,17]. It has been shown that CD49a enables direct selection and enrichment of human MSCs that express primitive stem cell markers [18]. Also, MSCs derived from embryonic stem cells appear
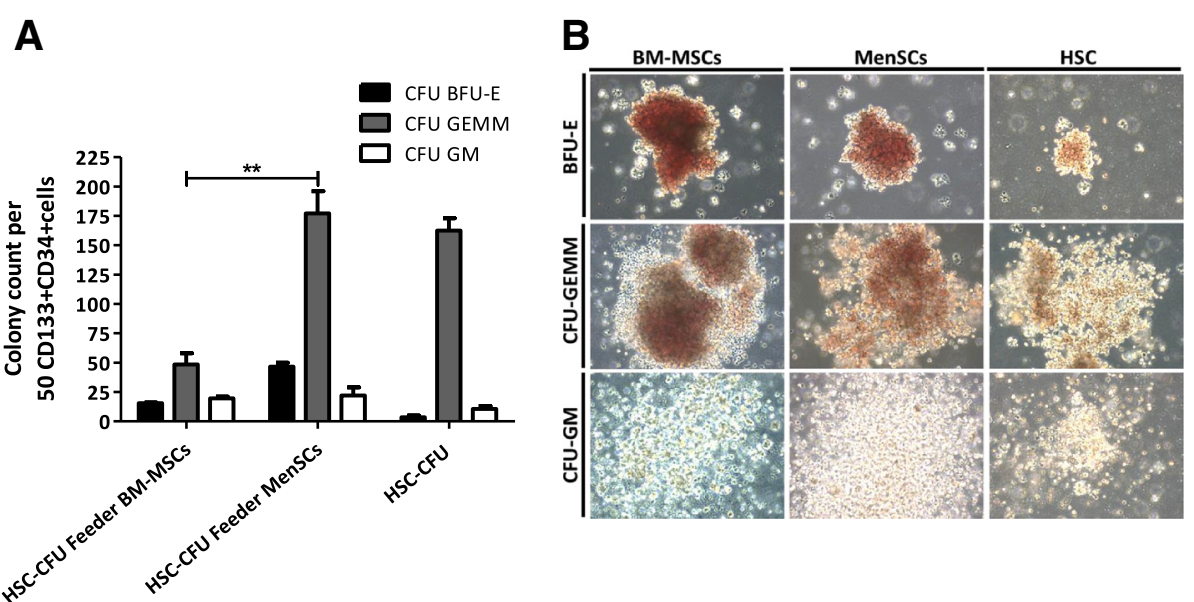

Figure 6 Ex vivo expansion of CD34 + CD133+ cells on MenSCs-feeder shows high potential of differentiation toward hematopoietic cells. In order to evaluate the progenitors of the hematopoietic lineage, CFU assay was performed by plating a single cell suspension in a semi-solid MethoCult ${ }^{\text {TM }}$ media. At 14 days post-culture, colony types derived from the initial equivalent of 50 cord blood HSC was classified and counted according to morphological criteria. (A) Total number of CFU-GEMM, CFU-GM, and BFU-E. Expansion of HSCs on the MenSCs-feeder have a higher capacity to generate CFU-GEMM compared to the other culture conditions. Values are expressed as mean \pm SE. ( $\left.{ }^{* *} P \leq 0.01\right)$. (B) Phase-contrast microscopy of representative CFU-GEMM, CFU-GM and BFU-E colonies. Images are representative of each condition. Original magnification x10. Data shown are representative of multiple replicates. BFU-E, burst-forming unit-erythroid; CFU-GEMM, colony-forming unit-granulocyte, erythroid, macrophage and megakaryocyte; CFU-GM, colony-forming unit-granulocyte and macrophage; HSCs, hematopoietic stem cells; SE, standard error. 
to express a high level of CD49a [19]. Furthermore, another difference in surface marker expression as reported by some groups, was the expression of embryonic markers, such as SSEA-4, in MenSCs that were absent on MSCs from other sources [3,20-22]. However, further results showed a different pattern, where MenSCs isolated and cultured under comparable conditions were negative for the same markers [2]. This raised the question whether these cells represent a more primitive progenitor stage than MSCs from other sources. For this reason, we have tested a larger panel of different embryonic stem (ES) markers including hTRA-1-60, SSEA3 and SSEA4 that were shown to be positive in MSCs isolated from fetal umbilical cords [23]. In our hands, none of these ES markers showed detectable expression on MSCs from the menstrual fluid or BM. Since no epidemiological study is available to date, it is unclear if the discrepancies in the expression of SSEA4, for example, are influenced by age, contraceptive treatments or other environmental factors.

As also demonstrated by others [2,5], the growth kinetics and clonality of MenSCs were significantly higher and these cells could be propagated for longer periods in culture without detectable changes in their proliferation rate. Nevertheless, the proliferation rate is not considered the sole criteria determining a higher abundance of MSCs in one cell source compared with others. The absolute and relative numbers of MSCs and the yield that can be isolated from a single donation are also essential. For this, we have first determined the frequency of CFU-F in menstrual fluid-derived MSCs by serial dilution. Considering the previously estimated number of CFU-F obtained from the bone marrow (about 0.001 to $0.01 \%$ ), cells isolated from the menstrual fluid yielded a two to four fold higher progenitor frequency, ranging from $0.004 \%$ to $0.02 \%$. Considering the average number of mononuclear cells that can be isolated from a single menstrual collection, the estimated absolute number of cells with clonogenic potential is 600 cells $/ \mathrm{ml}$ (see Additional file 3: Figure S3). To calculate the total number of progenitor cells that can be harvested from the same donor, the number of cells obtained from a single donation should be multiplied by the number of periodic samples obtained from the same donor. The results presented here point to the presence of higher relative and absolute numbers of MSCs in menstrual fluids compared to the bone marrow. It has also been shown that cells displaying a high CFU-F potential are obtained mostly from a CD49a + population [24]. As mentioned previously, MenSCs exhibited a higher expression of $\mathrm{CD} 49 \mathrm{a}$ + that could also account for the increased number of CFU-Fs, when similar cell dilution values were used. This attribute is meaningful in relation to their clinical use, since shorter culture times and larger expansion rates can hasten the harvest of the stem cell doses usually required for clinical use (around $1 \times 10^{6} / \mathrm{kg}$ ). The injection of cells at early passages could help avoid reaching the exhaustion of the stem cell properties or senescence that might limit therapeutic effect in patients.

We have also compared the tri-lineage differentiation potential, where MenSCs exhibited a lower adipogenic potential when compared to BM-MSCs. However, it will be interesting to assess more thoroughly the plasticity of these cells in the same comparison scheme, in the differentiation of other lineages. While no such head-to-head comparisons exist, Meng et al. showed that these cells can differentiate into nine lineages including cardiomyocytic, neurocytic, myocytic, endothelial, pancreatic, and hepatic [2]. Since cell migration is considered one of the mechanisms behind the MSCs-induced physiological effects in tissue repair, it was essential to understand their capacity to migrate to damaged tissues using an in vitro migration assay. MenSCs showed a higher migration potential with respect to BM-MSCs, where a complete wound closure was reached within 24 hours. While the migration capacity of MSCs is under the control of a large range of receptors and chemokines, and their subsequent homing to injured tissues may depend on the systemic and local inflammatory state, further in vivo studies are required to fully address this property.

To complete the functional study circle of the MenSCs, their angiogenic potential was assessed in vitro using HUVEC culture in normoxic and hypoxic conditions, and in vivo in a matrigel plug assay model. We demonstrated that the MenSCs CM was able to induce a significantly higher angiogenic effect on the HUVEC as evaluated by their reorganization and tube formation. We also noted that MenSCs were more responsive to hypoxic conditions, where a statistically significant increase of the secretion of the pro-angiogenic factor, VEGF, was detected. In line with these observations, the MenSCs were more efficient in inducing angiogenesis in the transplanted mice, where an eight fold increase in hemoglobin content was observed in implanted plugs containing MenSCs compared to BM-MSCs.

As MSCs participate in the bone marrow microenvironment by providing growth factors and matrix proteins, it has been demonstrated that they also provide an ex vivo support of the expansion of hematopoietic stem cells [11]. Here, we show for the first time, that MenSCs possess strong feeder properties as evidenced by a high expansion, maintenance of stemness and differentiation potential of co-cultured CD34 + CD133+ HSCs. Their support of HSCs cultures was superior to that of the BM-MSCs as a $3 \mathrm{X}$ expansion rate of the CD34 + CD133+ population as well as a higher number of early progenitor (CFU-GEMM) colonies was observed. Furthermore, we determined that the stromal support of the proliferation of CD34 + CD133+ cells on both MenSCs and BM-MSCs is cell contact dependent. To claim that MenSCs are the best source for the expansion of HSCs, one should also compare them to other MSC 
sources used as feeders. Since Klein et al. showed that the comparison of MSCs from bone marrow with MSCs from cord blood and amniotic fluid showed no significant difference in the expansion of HSCs [25], one can assume that MenSCs is an important source to consider for the ex vivo expansion of HSCs for therapeutic use, where the number of transplanted cells has been an important limitation for the success of the treatment [25]. All the comparative work described here was done using BM-MSC and MenSCs from different donors. It is important to take into consideration donor variation which is a bottleneck for standardization in such studies, as even demographically matched donors can also present different MSC performance as shown in Additional file 5: Figure S5. The ideal situation would be to perform this comparative study using different MSCs sources from the same donors; for this to happen, practical and possible ethical hurdles need to be overcome.

\section{Conclusions}

Whereas a variety of different tissue origins for the isolation of MSCs have been described, the menstrual fluid source may present a large number of advantages. These include being devoid from any ethical controversy, non-invasive and available for their isolation. More importantly, MenSCs possess increased proliferative, migration and pro-angiogenic capacity in vitro (especially under hypoxic conditions) and in animal models, in comparison to MSC populations obtained from bone marrow tissues. They also showed a significant stromal cell support for the expansion of HSCs. Therefore, in contrast to the limitations of bone marrow or other one-time harvest sources, such as placental tissues, MenSCs can be obtained repetitively and expanded to the larger quantities required for their therapeutic application. Taking into account all the advantages mentioned above, clinical applications may be based on their regenerative capacity, but more likely on the abundance, frequency, and expansion potential of these cells. However, it remains to determine which specific application is more appropriate for this cell source. It is important to stay away from the presumption that one source of stem cells is superior to other sources in all the existing cell based-therapies. Finally, we present data supporting the MenSCs as a very promising stem cell population. Whether these differences in comparison with BM-MSCs will have significant clinical impact on their functions as adult stem cells for regenerative medicine remains to be investigated.

\section{Additional files}

Additional file 1: Figure S1. MenSCs display a stable expression of different stem markers during long-term culture. To evaluate whether in vitro expansion influences the immunophenotype stability of MenSCs, cells were maintained in long-term culture and analyzed comparatively at early (P3 to 6) and late (P12 to 14) culture passages. FACS analysis showed that MenSCs (orange filled histograms) and BM-MSCs (green filled histograms) at early (soft colors) and late (dark colors) culture passages display the same pattern expression of stem cell-like phenotypic markers.

Additional file 2: Figure S2. MenSCs show a stable proliferation rate over long-term cultures. In order to evaluate the stability of the MenSCs and BM-MSCs proliferation in long-term cultures, proliferation assays were performed at early (P3 to 6) and late (P12 to 14) culture passages. No statistical differences were observed when comparing cell proliferation at early versus late passages in both MSCs sources.

Additional file 3: Figure S3. Limiting dilution analysis of MenSCs defines the frequency of progenitors cells. Mononuclear cells obtained after the ficoll centrifugation of the menstrual blood were resuspended in DMEM and plated at a density of 100, 1,000,10,000, and 100,000 nucleated cells/cm2. Media were changed the next day to wash non adherent cells. The frequency of progenitors was calculated by counting the number of CFU-F in each determined dilution and following the extreme limiting dilution analysis (ELDA) method for comparing depleted and enriched populations in stem cells. The absolute number of progenitor cells $/ \mathrm{ml}$ of menstrual blood was obtained by multiplying the frequency of progenitors with the number of total mononuclear cells isolated from $1 \mathrm{ml}$ of menstrual blood post-Ficoll isolation.

Additional file 4: Figure S4. MenSCs show a stable colony forming unit potential (CFU-F) in long-term cultures. To evaluate whether long-term expansion affects the MenSCs CFU potential, cells were maintained in culture for multiple passages and analyzed comparatively at early (P3 to 6) and late (P12 to 14) culture passages. Statistical analysis reveals that no significant variation in the CFU potential was observed between early and late passages (left panel). Photographs are representative of the CFU at day 12 (right panel).

Additional file 5: Figure S5. MenSCs variability in their properties are donor dependent. Ten samples from different donors were isolated and serial dilutions of a defined number of cells were cultured and their potential for the formation of CFU was evaluated. (A) Total number of CFU colony; (B) Representative images of CFU colony.

\section{Abbreviations}

bFGF: basic fibroblast growth factor; BFU-E: burst-forming unit-erythroid; BM: bone marrow; BM-MSCs: bone marrow derived mesenchymal stem cells; BSA: bovine serum albumin; CFU-F: fibroblast colony-forming units; CFU-GEMM: colony-forming unit-granulocyte, erythroid, macrophage and megakaryocyte; CFU-GM: colony-forming unit-granulocyte and macrophage; CM: conditioned media; DMEM: (Dulbecco's) modified Eagle's medium; ELDA: extreme limiting dilution analysis; ELISA: enzyme-linked immunosorbent assay; FACS: fluorescence-activated cell sorting; HPCs: hematopoietic progenitor cells; HSCs: hematopoietic stem cells: HUVEC: human umbilical vein endothelial cells; MenSCs: menstrual derived stem cells; MSCs: mesenchymal stem cells; NSG: Nod scid gamma mice; OC: osteocalcin; PBS: phosphate-buffered saline; P/S: penicillin/streptomycin; TW: transwell; VEGF: vascular endothelial growth factor.

\section{Competing interests}

Maroun Khoury is the CSO of Cells for Cells and REGENERO, Francisca Alcayaga-Miranda, Jimena Cuenca, Ainoa Fernandez and Carolina Aguila-Díaz received stipends from Cells for Cells. The other authors declare that they have no competing interests.

\section{Authors' contributions}

FAM, JC, PLC and MK contributed to conception and design, acquisition of data, and the analysis and interpretation of data. FAM, CAD and AF were involved in performing the experiments and data analysis. FAM and MK prepared the manuscript. FEF assisted in the interpretation of the data and did substantial contribution in the discussion of the results. FEF and MK critically revised the manuscript. All authors read and approved the final manuscript.

\section{Acknowledgments}

We thank Samy Silva and CCHEN for technical assistance in the irradiation of the cells; Enrique Montiel and Diego Seguel for excellent technical assistance in cell culture; Dr. Jorge Bartolucci and Dr. Hugo Azocar for providing the 
primary bone marrow cells; Macarena Ocaña and Claudia Rubí for help in animal model experiments; Paz Gonzalez for technical assistance in angiogenesis experiments; and members of the C4C lab for support and helpful discussion. This work was funded by the Chilean National Commission for Scientific and Technological Investigation (CONICYT), FONDEF IDeA Program, grant number CA12i10349 and Cells for Cells S.A.

\section{Author details}

'Laboratory of Nano-Regenerative Medicine, Faculty of Medicine, Universidad de Los Andes, Santiago, Chile. ${ }^{2}$ Cells for Cells, Santiago, Chile. ${ }^{3}$ Inserm, U844, Montpellier F-34295, France.

Received: 22 July 2014 Revised: 20 February 2015 Accepted: 20 February 2015 Published online: 17 March 2015

\section{References}

1. Akimoto K, Kimura K, Nagano M, Takano S, To'a Salazar G, Yamashita T, et al. Umbilical cord blood-derived mesenchymal stem cells inhibit, but adipose tissue-derived mesenchymal stem cells promote, glioblastoma multiforme proliferation. Stem Cells Dev. 2013;22:1370-86.

2. Meng X, Ichim TE, Zhong J, Rogers A, Yin Z, Jackson J, et al. Endometrial regenerative cells: a novel stem cell population. J Transl Med. 2007;5:57.

3. Cui CH, Uyama T, Miyado K, Terai M, Kyo S, Kiyono T, et al. Menstrual bloodderived cells confer human dystrophin expression in the murine model of Duchenne muscular dystrophy via cell fusion and myogenic transdifferentiation. Mol Biol Cell. 2007;18:1586-94.

4. Hida N, Nishiyama N, Miyoshi S, Kira S, Segawa K, Uyama T, et al. Novel cardiac precursor-like cells from human menstrual blood-derived mesenchymal cells. Stem Cells. 2008;26:1695-704.

5. Patel AN, Park E, Kuzman M, Benetti F, Silva FJ, Allickson JG. Multipotent menstrual blood stromal stem cells: isolation, characterization, and differentiation. Cell Transplant. 2008;17:303-11.

6. Borlongan CV, Kaneko Y, Maki M, Yu SJ, Ali M, Allickson JG, et al. Menstrual blood cells display stem cell-like phenotypic markers and exert neuroprotection following transplantation in experimental stroke. Stem Cells Dev. 2010;19:439-52.

7. Patel AN, Silva F. Menstrual blood stromal cells: the potential for regenerative medicine. Regen Med. 2008;3:443-4.

8. Nagaya N, Kangawa K, Itoh T, Iwase T, Murakami S, Miyahara Y, et al. Transplantation of mesenchymal stem cells improves cardiac function in a rat model of dilated cardiomyopathy. Circulation. 2005;112:1128-35.

9. Pittenger MF, Martin BJ. Mesenchymal stem cells and their potential as cardiac therapeutics. Circ Res. 2004;95:9-20.

10. Poloni A, Maurizi G, Leoni P, Serrani F, Mancini S, Frontini A, et al. Human dedifferentiated adipocytes show similar properties to bone marrow-derived mesenchymal stem cells. Stem Cells. 2012;30:965-74.

11. Khoury M, Drake A, Chen Q, Dong D, Leskov I, Fragoso MF, et al. Mesenchymal stem cells secreting angiopoietin-like-5 support efficient expansion of human hematopoietic stem cells without compromising their repopulating potential. Stem Cells Dev. 2011;20:1371-81.

12. Khanjani S, Khanmohammadi M, Zarnani AH, Akhondi MM, Ahani A, Ghaempanah Z, et al. Comparative evaluation of differentiation potential of menstrual blood- versus bone marrow- derived stem cells into hepatocyte-like cells. PLoS One. 2014;9:e86075.

13. Bockeria L, Bogin V, Bockeria O, Le T, Alekyan B, Woods EJ, et al. Endometrial regenerative cells for treatment of heart failure: a new stem cell enters the clinic. J Transl Med. 2013;11:56.

14. Hu Y, Smyth GK. ELDA: extreme limiting dilution analysis for comparing depleted and enriched populations in stem cell and other assays. J Immunol Methods. 2009;347:70-8.

15. Dominici M, Le Blanc K, Mueller I, Slaper-Cortenbach I, Marini F, Krause D, et al. Minimal criteria for defining multipotent mesenchymal stromal cells. The International Society for Cellular Therapy position statement. Cytotherapy. 2006:8:315-7.

16. Muller WA. Mechanisms of transendothelial migration of leukocytes. Circ Res. 2009;105:223-30.

17. Sandoval F, Terme M, Nizard M, Badoual C, Bureau MF, Freyburger $L$, et al. Mucosal imprinting of vaccine-induced CD8(+) T cells is crucial to inhibit the growth of mucosal tumors. Sci Transl Med. 2013;5:172ra120.

18. Gindraux F, Selmani Z, Obert L, Davani S, Tiberghien P, Herve P, et al. Human and rodent bone marrow mesenchymal stem cells that express primitive stem cell markers can be directly enriched by using the CD49a molecule. Cell Tissue Res. 2007;327:471-83.

19. Vemuri MC, Chase LG, Rao MS. Mesenchymal stem cell assays and applications. Methods Mol Biol. 2011;698:3-8.

20. Allickson J, Xiang C. Human adult stem cells from menstrual blood and endometrial tissue. J Zhejiang Univ Sci B. 2012;13:419-20.

21. Allickson JG, Sanchez A, Yefimenko N, Borlongan CV, Sanberg PR. Recent studies assessing the proliferative capability of a novel adult stem cell identified in menstrual blood. Open Stem Cell J. 2011;3:4-10.

22. Mou XZ, Lin J, Chen JY, Li YF, Wu XX, Xiang BY, et al. Menstrual blood-derived mesenchymal stem cells differentiate into functional hepatocyte-like cells. J Zhejiang Univ Sci B. 2013;14:961-72.

23. Jo CH, Kim OS, Park EY, Kim BJ, Lee JH, Kang SB, et al. Fetal mesenchymal stem cells derived from human umbilical cord sustain primitive characteristics during extensive expansion. Cell Tissue Res. 2008;334:423-33.

24. Deschaseaux F, Gindraux F, Saadi R, Obert L, Chalmers D, Herve P. Direct selection of human bone marrow mesenchymal stem cells using an anti-CD49a antibody reveals their CD45med, low phenotype. Br J Haematol. 2003;122:506-17.

25. Klein C, Strobel J, Zingsem J, Richter RH, Goecke TW, Beckmann MW, et al, Ex vivo expansion of hematopoietic stem- and progenitor cells from cord blood in coculture with mesenchymal stroma cells from amnion, chorion, Wharton's jelly, amniotic fluid, cord blood, and bone marrow. Tissue Eng Part A. 2013;19:2577-85.

\section{Submit your next manuscript to BioMed Central and take full advantage of:}

- Convenient online submission

- Thorough peer review

- No space constraints or color figure charges

- Immediate publication on acceptance

- Inclusion in PubMed, CAS, Scopus and Google Scholar

- Research which is freely available for redistribution 